УДК 616.314-089.23

\title{
БАЗОВЫЕ КОМПОНЕНТЫ СТОМАТОЛОГИЧЕСКИХ САD/САМ СИСТЕМ. АНАЛИЗ СКАНИРУЮЩИХ УСТРОЙСТВ, ПРИМЕНЯЕМЫХ НА ОРТОПЕДИЧЕСКОМ ПРИЕМЕ. ПРЕИМУЩЕСТВА И НЕДОСТАТКИ ЭКСПЛУАТАЦИИ ОБОРУДОВАНИЯ ЦИФРОВОЙ СТОМАТОЛОГИИ
}

\section{Пашкевич Владислав Дмитриевич}

студент

Научные руководители: Абаджян Лерник Владимирович ассистент каф. пропедевтики стоматологических заболеваний

Ерёмин Олег Вячеславович

д.м.н., доцент зав. каф. пропедевтики стоматологических заболеваний Кобзева Юлия Александровна к.М.н., доцент

зав. учебной частью каф. пропедевтики стоматологических заболеваний ФГБОУ ВО «Саратовский ГМУ им. В.И. Разумовского Минздрава России»

Аннотация: Появление CAD/CAM систем стало важным этапом развития стоматологии, позволившим упростить этапы изготовления зубных протезов. Чем раньше будущий квалифицированный специалист узнает о возможностях цифровой стоматологии, тем быстрее и с большим успехом в перспективе он сможет применять данную технологию на практике для осуществления высокотехнологичного и качественного лечения.

Цель исследования - создать материал для осведомления студентов, ординаторов и практикующих врачей об устройстве стоматологических CAD/CAM систем, ознакомить с преимуществами и недостатками применения технологий цифровой стоматологии, дополнительно акцентировать внимание на анализе сканирующих устройств. Научная новизна работы заключается в осуществлённом анкетировании среди 137 студентов и ординаторов стоматологического факультета «ФГБОУ ВО Саратовский ГМУ им. В.И. Разумовского Минздрава России», которое выявило низкую осведомленность обучающихся в вопросах цифровой стоматологии. В результате проведенного исследования был создан 295 


\title{
ЦИФРОВИЗАЦИЯ КАК НОВАЯ ПАРАДИГМА РАЗВИТИЯ: ВЫЗОВЫ, ВОЗМОЖНОСТИ И ПЕРСПЕКТИВЫ
}

теоретический материал, подробно отражающий устройство и основные компоненты стоматологических $\mathrm{CAD} / \mathrm{CAM}$ систем, преимущества и недостатки технологий цифровой стоматологии, произведён анализ сканирующих устройств разных видов.

Ключевые слова: CAD/CAM, анкетирование, цифровая стоматология, зубной протез, лабораторный и клинический сканер, программное обеспечение, субтрактивное и аддитивное производство, расходные материалы, преимущества и недостатки.

\section{BASIC COMPONENTS OF DENTAL CAD / CAM SYSTEMS. ANALYSIS OF SCANNING DEVICES USED AT ORTHOPEDIC RECEPTION. ADVANTAGES AND DISADVANTAGES OF OPERATING DIGITAL DENTISTRY EQUIPMENT}

\author{
Pashkevich Vladislav Dmitrievich \\ Scientific supervisors: Abajyan Lernik Vladimirovich \\ Eremin Oleg Vyacheslavovich \\ Kobzeva Yulia Aleksandrovna
}

\begin{abstract}
The advent of CAD / CAM systems has become an important stage in the development of dentistry, which has made it possible to simplify the stages of manufacturing dentures. The sooner the future qualified specialist learns about the possibilities of digital dentistry, the faster and with greater success in the future he will be able to apply this technology in practice for high-tech and high-quality treatment.

The purpose of the study is to create material for informing students, residents and practitioners about the device of dental CAD/CAM systems, to familiarize them with the advantages and disadvantages of using digital dentistry technologies, and to additionally focus on the analysis of scanning devices. The scientific novelty of the work lies in the survey conducted among 137 students and residents of the Faculty of Dentistry "Saratov State Medical University named after V. I. Razumovsky of the Ministry of Health of Russia", which revealed a low awareness of students in digital dentistry. As a result of the research, a theoretical material was created that reflects in detail the device and the main components of dental CAD/CAM systems, the advantages and disadvantages of digital dentistry technologies, and the analysis of scanning devices of various types was performed.
\end{abstract}




\section{ЦИФРОВИЗАЦИЯ КАК НОВАЯ ПАРАДИГМА РАЗВИТИЯ:

Key words: CAD/CAM, survey, digital dentistry, dental prosthesis, laboratory and clinical scanner, software, subtractive and additive manufacturing, consumables, advantages and disadvantages.

Чтобы выяснить заинтересованность обучающихся в вопросах цифровой стоматологии было проведено анкетирование среди 137 студентов и ординаторов стоматологического факультета «ФГБОУ ВО Саратовский ГМУ им. В.И. Разумовского Минздрава России».

Результаты опроса говорят, что $41 \%$ студентов и ординаторов не имеют ни малейшего представления о СAD/CAM системах в стоматологии, а $46 \%$ людей слышали, но при попытках узнать больше столкнулись с проблемой поиска литературы (рис. 1). Всего лишь 13\% людей хорошо разбираются в данной сфере, но ответили не на все вопросы правильно.

\footnotetext{
Имеется ли у вас представление о CAD/CAM системах в стоматологии?

137 ответов
}

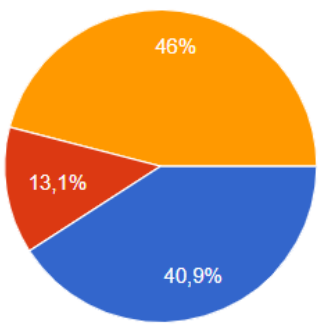

Не имею ни малейшего

представления

Хорошо знаком и разбираюсь в этом

Слышал, но при желании разобраться

в вопросе, столкнулся с проблемой

поиска литературы

\section{Рис. 1. Степень осведомленности обучающихся в вопросе цифровой стоматологии}

CAD/CAM - это современная цифровая методика, которая автоматизирует процесс создания зубных протезов: заготовка моделируется в электронной программе и фрезеруется на станке с числовым программным управлением.

Вне зависимости от производителя любая современная CAD/CAM система включает следующие элементы:

1. Сканер;

2. Программное обеспечение;

3. Технология производства (фрезерная установка, 3D - принтер).

Система может быть замкнутой или открытой. В замкнутых системах программное обеспечение работает только с фрезером и сканером компании 


\section{ЦИФРОВИЗАЦИЯ КАК НОВАЯ ПАРАДИГМА РАЗВИТИЯ: ВЫЗОВЫ, ВОЗМОЖНОСТИ И ПЕРСПЕКТИВЫ}

производителя. Открытая система позволяет взаимодействовать с другими технологиями и дополняться оборудованием от других производителей $[1$, c. 3].

\section{CAD/CAM сканер}

Это устройство, позволяющее перевести геометрию объекта в цифровые данные. Сканирование протезного ложа является первым этапом при изготовлении зубного протеза при помощи любой $\mathrm{CAD} / \mathrm{CAM}$ системы. По назначению выделяют клинические (рис. 2) и лабораторные (рис. 3) сканеры.

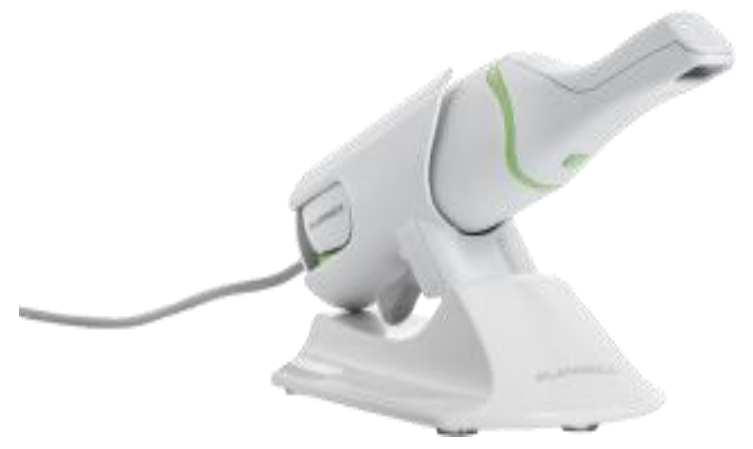

\section{Рис. 2. Клинический сканер}

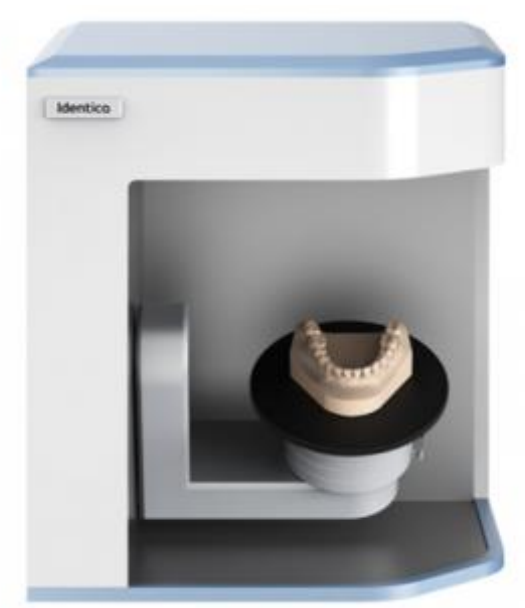

\section{Рис. 3. Лабораторный сканер}

В составе нашей анкеты был вопрос: «Какой сканер более точен в измерениях, лабораторный или клинический? Большинство студентов, ответили, что клинический сканер обладает более высокой точностью, что является неверным (рис. 4). Необходимо разобраться в причине этого заблуждения. 
Какой вид CAD/CAM сканера имеет более высокую точность?

136 ответов

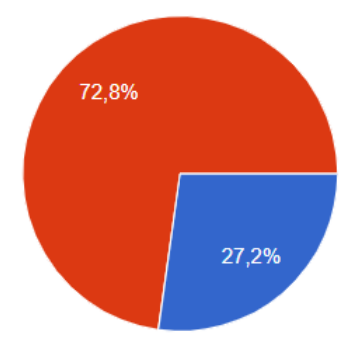

Лабораторный

Интраоральный

\section{Рис. 4. Вопрос с наибольшим количеством неправильных ответов}

Клинические (интраоральные сканеры) позволяют сканировать ротовую полость пациента напрямую, в режиме реального времени получать цифровые модели зубов. Вместо традиционных гипсовых слепков врач получает оцифрованные изображения в формате .STL.

Основные преимущества интраоральных сканеров [2, с. 15].:

1) Отсутствие затрат на слепочный материал и гипс;

2) Сканирующая камера портативна и имеет размеры немного больше зубной мануальной щётки;

3) Сканер воспроизводит реалистичное изображение актуальной ситуации в полости рта;

4) Возможно автоматическое или полуавтоматическое определение центральной окклюзии.

Отрицательные моменты при сканировании интраоральным сканером:

1) Меньшая видимость протезного ложа (особенно в пришеечной области);

2) Увеличенное время сканирования за счет тремора руки операциониста.

Лабораторные сканеры предназначены для сканирования гипсовых моделей, либо оттисков. Последние разработки позволяют осуществлять оцифровку моделей, загипсованных в артикуляторе.

Преимущества лабораторных сканеров [2, с. 16]:

1) Возможность перевода большого количества объектов в цифровую форму в течение короткого временного промежутка;

2) Лабораторные сканеры в отличие от клинических обладают более высокой точностью и скоростью сканирования за счет фиксированного фокусного расстояния и отсутствия смещений объекта сканирования. 
Недостатки [2, с. 16]:

1) Усадка оттискного материала;

2) Недостатки оттиска или гипсовой модели;

3) Расширение гипса.

При соблюдении правильной техники работы таких последствий можно избежать.

\section{Программное обеспечение}

Программное обеспечение позволяет редактировать полученный цифровой файл. Программа имеет определенный набор инструментов, позволяющих моделировать любые виды ортопедических конструкций.

Ортопедический модуль (рис. 5), является базовым во всех программах, но по мере развития технологий производители начали активно выпускать дополнительные ортодонтические (рис. 6) и хирургические модули (рис. 7). Особенно интересен дополнительный модуль «Smile Design» в программе ЕхоCAD, позволяющий моделировать будущую улыбку пациента (рис. 8).

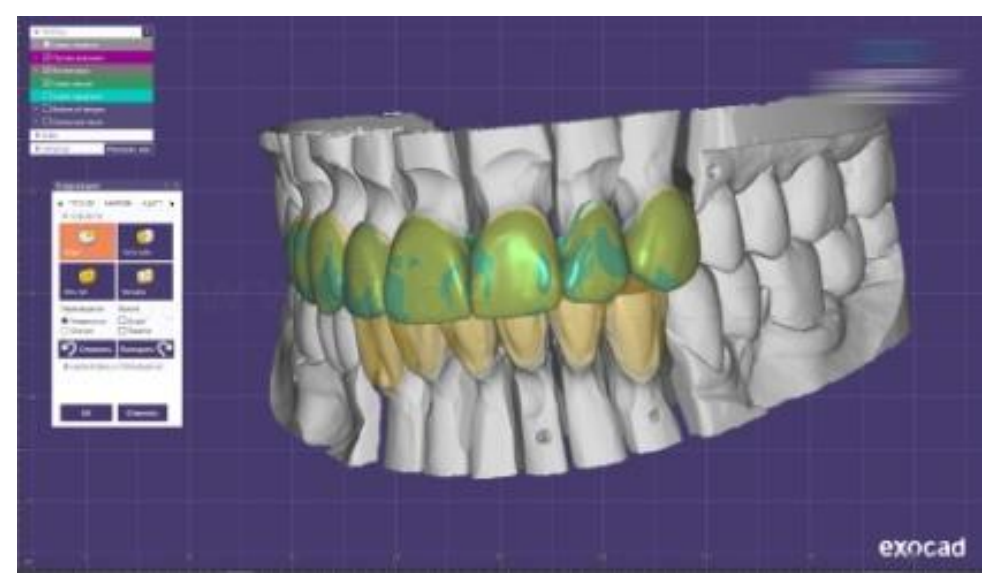

Рис. 5. Ортопедический модуль

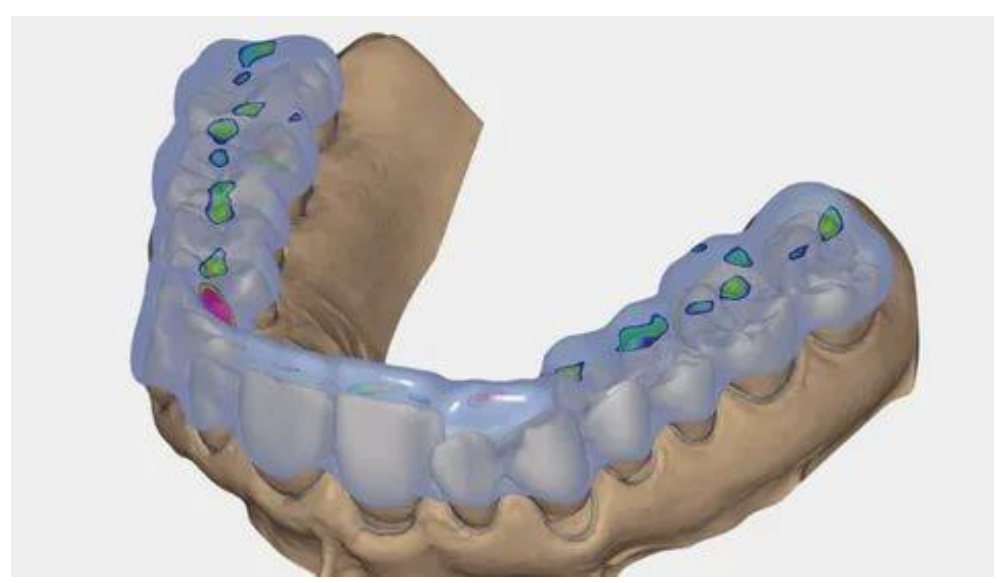

Рис. 6. Ортодонтический модуль

300 


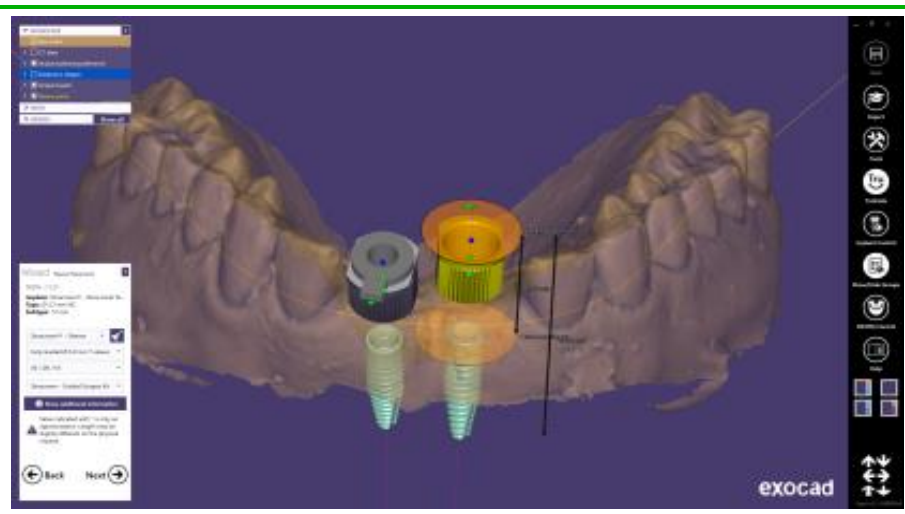

Рис. 7. Хирургический модуль

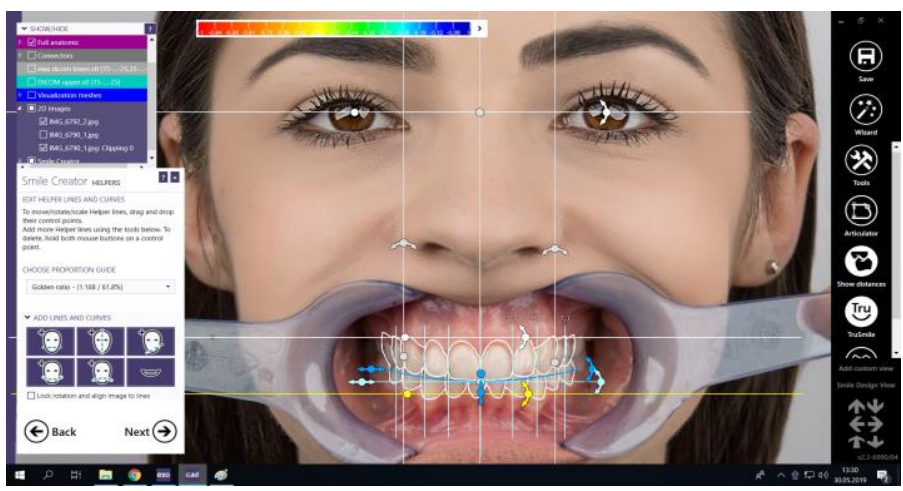

Рис. 8. Модуль «Smile Design»

CAD/CAM технология производства

Последним этапом работы с CAD/CAM системой является трансформирование цифровых данных в готовое изделие. Все технологии производства делятся на субтрактивные и аддитивные.

Изготовление зубного протеза по технологии CAD/CAM основано главным образом, вокруг процесса субтрактивного производства. Происходит фрезеровка объекта из заготовки материала на специальных станках (рис. 9), которые контролируются компьютерной программой.

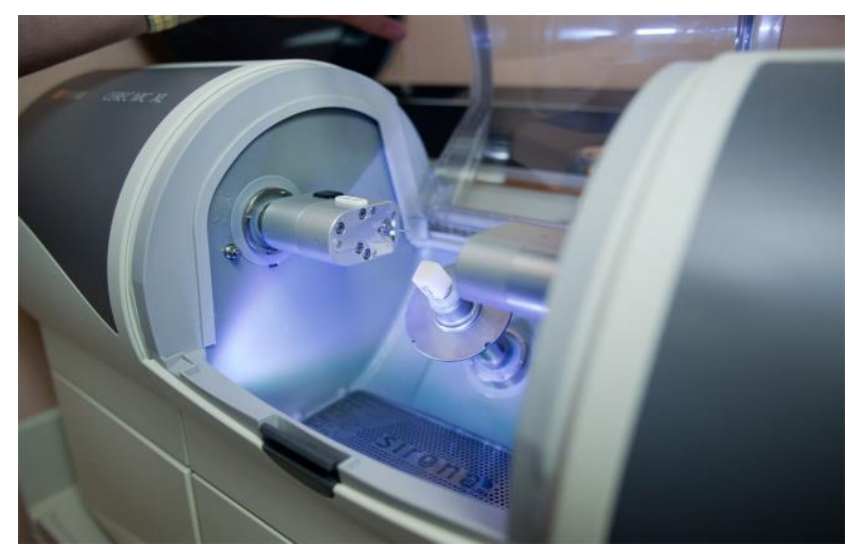

Рис. 9. CAD/CAM фрезер 


\section{ЦИФРОВИЗАЦИЯ КАК НОВАЯ ПАРАДИГМА РАЗВИТИЯ: ВЫЗОВЫ, ВОЗМОЖНОСТИ И ПЕРСПЕКТИВЫ}

Фрезерные станки могут иметь клиническое, либо лабораторное назначение. Они различаются в зависимости от материала для обработки, размера заготовок, мощности аппарата, количества осей для фрезерования.

Аддитивная технология основана на компьютерной обработке 3Dфайлов, после которой цифровой объект превращается в набор срезов определенной толщины (рис. 10). Каждый слой затем печатается один поверх другого, чтобы создать 3D-объект. При помощи такой технологии можно печатать различные виды протезов, большим успехом выделяется изготовление элайнеров таким способом (рис. 11).

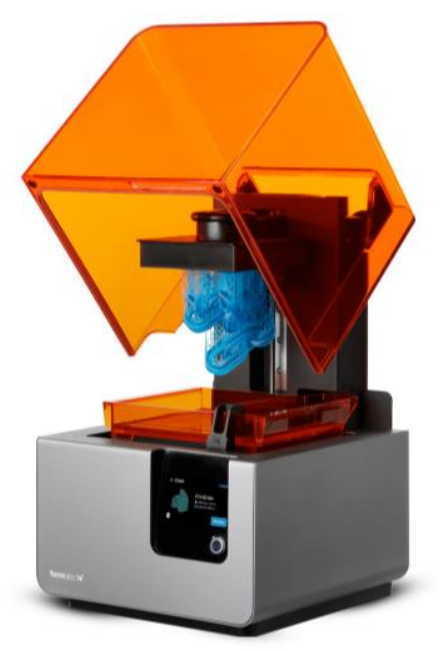

\section{Рис. 10. CAD/CAM 3-D принтер}

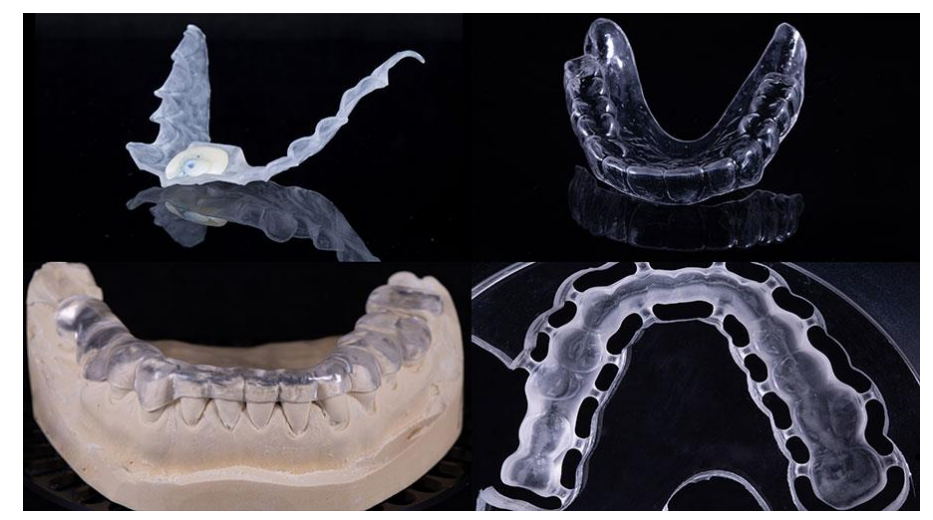

Рис. 11. Элайнеры, изготовленные на 3D-принтере

Основные расходные материалы, которые необходимы при работе с САD/САМ системой

1) Спрей для сканирования, позволяющий исключить глянцевый эффект при сканировании как на модели, так и в полости рта, он убирает блики, 


\section{ЦИФРОВИЗАЦИЯ КАК НОВАЯ ПАРАДИГМА РАЗВИТИЯ: ВЫЗОВЫ, ВОЗМОЖНОСТИ И ПЕРСПЕКТИВЫ}

формируя матовое покрытие. Точное объемное изображение можно получить, если нет искажений и бликов, которые могут воздействовать на луч сканера (рис. 12).

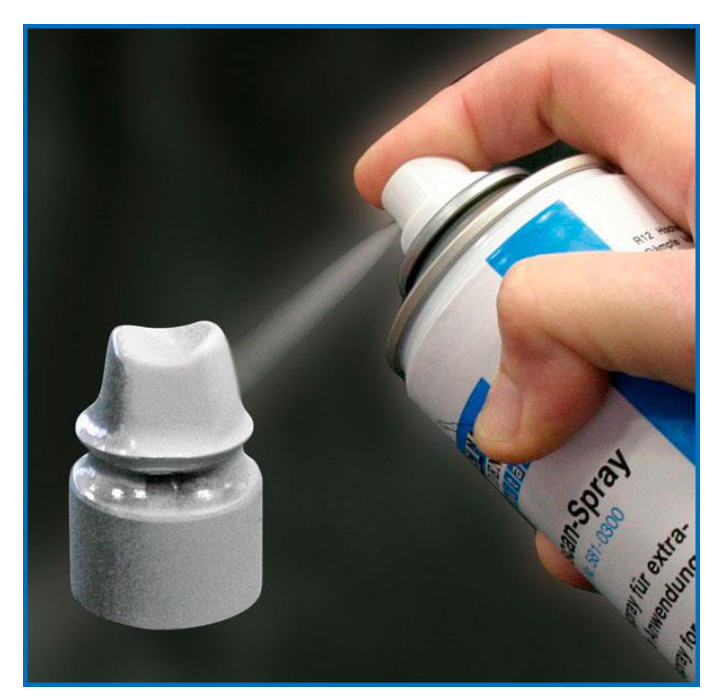

\section{Рис. 12. Спрей для сканирования}

2) Заготовки для фрезерования. Обычно производятся в форме дисков (рис. 13), либо кубических блоков (рис. 14). Тип заготовки зависит от вида фрезерного станка. Кубические блоки обычно предназначены для клинических фрезеров, при изготовления единичных конструкций. Диски применяются в более продвинутых лабораторных фрезерных станках, с их помощью можно изготавливать более протяженные конструкции.

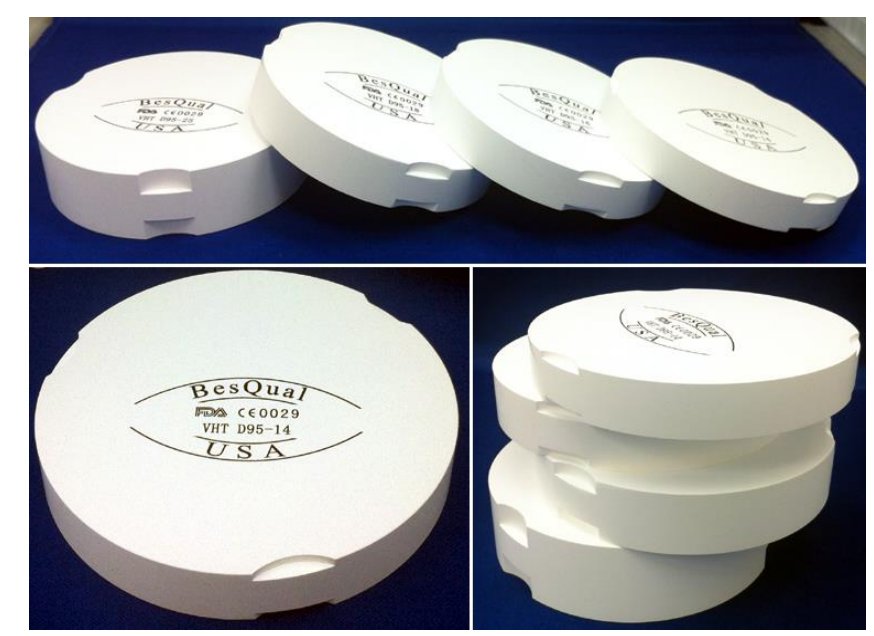

Рис. 13. Заготовки для фрезерования в форме дисков 


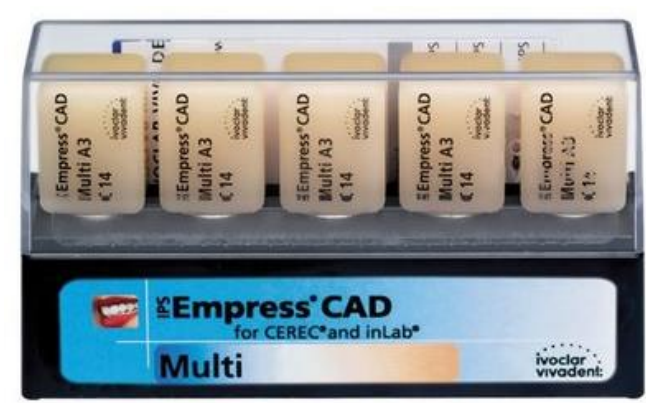

\section{Рис. 14. Кубические заготовки для фрезерования}

3) Не менее важным расходным материалом являются фрезы, которые необходимы для сошлифовывания из заготовок материала готового протеза (рис. 15).

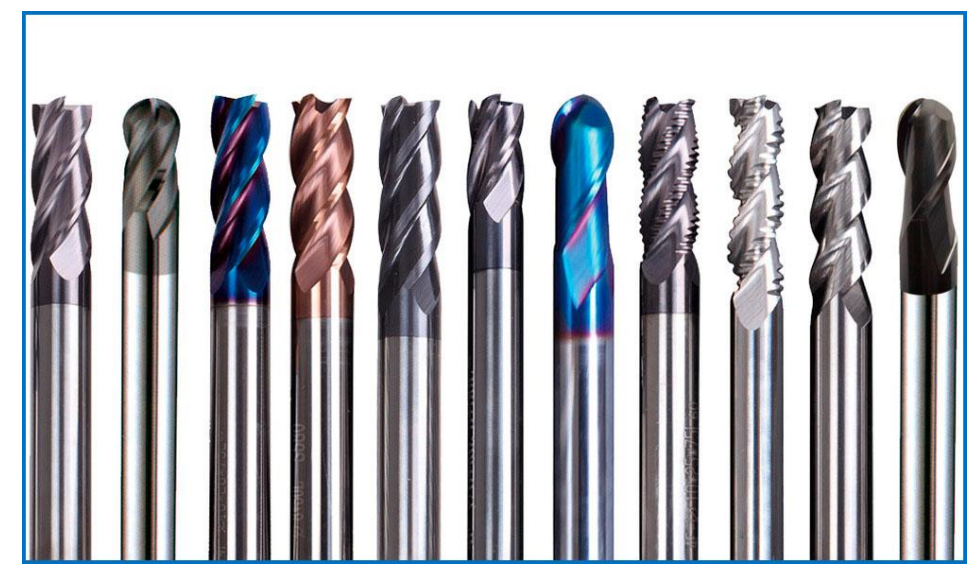

Рис. 15. Фрезы

Фрезы в основном сделаны из твердосплавного материала, могут иметь алмазное напыление. Линейки фрез разделены по типу обрабатываемого материала и диаметру. Кроме того, выделяют торцевые и сферические фрезы. Первый вариант используется при обработке уступов, пазов, сложных поверхностей. Сферические концевые инструменты необходимы для удаления излишков материала на участках сложной формы. Рабочая поверхность срезает слои по всей протяженности, что позволяет создавать геометрию, в точности повторяющую естественную форму компонентов ротовой полости.

Преимущества и недостатки CAD/CAM систем

Преимущества САD/САМ систем

1. Эксплуатация CAD/CAM систем дает возможность быстро осуществлять протезирование, при необходимости в одно посещение, что повышает комфорт пациента; 


\section{ЦИФРОВИЗАЦИЯ КАК НОВАЯ ПАРАДИГМА РАЗВИТИЯ: ВЫЗОВЫ, ВОЗМОЖНОСТИ И ПЕРСПЕКТИВЫ}

2. Эксплуатация клинических и зуботехнических CAD/CAM систем позволяет снизить нагрузку на зубного техника, зачастую имеющего избыток работы, что позволяет ему сосредоточиться на более сложной и объемной работе;

3. Использование системы, включающей интраоральный сканер, позволяет избавиться от некоторых расходных материалов (слепочная масса, гипс) и сэкономить время на создании моделей, что ускоряет весь процесс;

4. Готовый скан ротовой полости пациента представлен цифровым файлом в формате .STL, который может моментально отправляться в зуботехническую лабораторию, как самой клиники, так и в любой точке города, где сразу же может начаться этап программной обработки и создания готовой конструкции;

5. При использовании аддитивной технологии производства отсутствуют отходы;

6. Использование CAD/CAM систем на ортопедическом приеме гарантирует высокую эстетику, так как программное создание модели протеза позволяет воспроизводить анатомию зубов в мельчайших подробностях;

7. Многообразие используемых материалов (воск, пластмасса, акрил, диоксид циркония, керамика, композиты, металлы и сплавы и др.).

Недостатки следуюшие:

1. Дороговизна. К примеру, полный комплект CAD/CAM системы в сборе может обойтись в практически 6 млн.р.;

2. Субтрактивная методика за счёт особенностей производства оставляет много отходов материала, количество которых может многократно превышать объем самого готового изделия.

Заключение

Анализ ответов после проведенного анкетирования показал низкую осведомленность студентов и ординаторов в вопросах цифровой стоматологии. Обучающиеся сталкивались с трудностями в поиске литературы. Исходя из этого, был создан краткий материал для осведомления интересующихся лиц об устройстве стоматологических CAD/CAM систем.

В представленном материале акцентировано внимание на основных компонентах и их тесном взаимодействии, проведен тщательный анализ 3Dсканеров, кроме этого отражены преимущества и недостатки технологий цифровой стоматологии. 


\section{ЦИФРОВИЗАЦИЯ КАК НОВАЯ ПАРАДИГМА РАЗВИТИЯ: ВЫЗОВЫ, ВОЗМОЖНОСТИ И ПЕРСПЕКТИВЫ}

\section{Список литературы}

1. Наумович C.C., Разоренов А.Н., CAD/Саm системы в стоматологии: современное состояние и перспективы развития // Современная стоматология. - 2016. - №4 (65);

2. CAD/CAM-системы в стоматологии: учебное пособие / С.И. Абакаров, А.С. Баландина, Д.В. Сорокин, К.С. Аджиев, С.С. Абакарова, Д.С. Арутюнов; ФГБОУ ДПО РМАНПО, 2016. - 96 с.;

3. Ряховский А.Н. Цифровая стоматология. - М., 2010. - 282 с.;

4. Alghazzawi IF H J. Prosthodont. Res. - 2016. - Vol.60, N2. - P.72-84.;

5. Beguma Z , Chhedat P. //Int. J. Comput. Dent. - 2014.-Vol.17, N4.-P.297306 ;

6. Komine F, Blatz M.B., Matsumura H. / / J Oral. Sci. - 2010. - Vol.52, N4. P.531-539.;

7. Liu P.P. //Compend Contin. Educ. Dent. - 2005. - Vol.26, N7. - P.507508.;

8. LimaJ.M., AnamiL.C., Araujo P.M., PavanellC.A. // J. Prosthodont. 2014. - Vol.23, N7. - P.588-591.;

9. NayarS., Bhuminathan S., Bhat W.M. //J. Pharm. Bioallied. Sci. - 2015. Vol.7 (Suppl.1). - P.216-219.;

10. Patel N. //Compend Contin, Educ. Dent. - 2014. - Vol.35, N10.-P.739746.;

11. Torabi K., Farjood E, Hamedanib Sh. //X Dent. (Shiraz).- 2015,- Vol.16. P. 1 - 9.;

12. Одонтопрепарирование при лечении винирами и керамическими коронками / С.Д.Арутюнов, А.И.Лебеденко, Т.Э. Глебова, И.Ю. Лебеденко М.: Молодая гвардия, 2008. - 136 с.;

13. Андреас Эндер, Вернер X. Мёрманн. CEREC 3D DESIGN. Компьютерное конструирование и изготовление (CAD/CAM) цельнокерамических вкладок, коронок и виниров. Компьютерное конструирование и изготовление. - Цюрих, 2005 г. -258 с. 